\title{
Aurora A Kinase Function at Kinetochores
}

\author{
JENNIFER G. DeLuCA \\ Department of Biochemistry and Molecular Biology, Colorado State University, Fort Collins, \\ Colorado 80523-1870 \\ Correspondence: jdeluca@colostate.edu
}

\begin{abstract}
One of the most important regulatory aspects of chromosome segregation is the ability of kinetochores to precisely control their attachment strength to spindle microtubules. Central to this regulation is Aurora B, a mitotic kinase that phosphorylates kinetochore substrates to promote microtubule turnover. A critical target of Aurora B is the kinetochore protein Ndc80/ $\mathrm{Hec} 1$, which is a component of the NDC80 complex, the primary force-transducing link between kinetochores and microtubules. Although Aurora B is regarded as the "master regulator" of kinetochore-microtubule attachment, it is becoming clear that this kinase is not solely responsible for phosphorylating Hec1 and other kinetochore substrates to facilitate microtubule turnover. In particular, there is growing evidence that Aurora A kinase, whose activities at spindle poles have been extensively described, has additional roles at kinetochores in regulating the kinetochore-microtubule interface.
\end{abstract}

\section{REGULATION OF KINETOCHORE- MICROTUBULE ATTACHMENT STABILITY}

Kinetochores are large protein structures assembled on centromeric chromatin that power and regulate chromosome segregation during mitosis. These orchestrators of mitosis physically connect chromosomes to spindle microtubules and transduce forces through the connections to align and segregate chromosomes. Successful mitosis requires that kinetochores precisely regulate their attachment strength to microtubules. In early mitosis, kinetochores ensure that attachments are labile and short-lived so that improper attachments are corrected, whereas in late mitosis, kinetochores form stable, persistent attachments to microtubules so that forces can be generated to drive chromosome movements and to silence the spindle assembly checkpoint (Funabiki and Wynne 2013; Godek et al. 2015; Lampson and Grishchuk 2017; Musacchio and Desai 2017). Central to this regulation is Aurora B, a mitotic kinase that phosphorylates kinetochore substrates to promote microtubule turnover (Carmena et al. 2012; Krenn and Musacchio 2015; Hindriksen et al. 2017). A key Aurora $\mathrm{B}$ target involved in this regulation is the $\mathrm{Hec} 1$ protein of the kinetochore-associated NDC80 complex, a primary contributor to the generation of stable, end-on attachments to spindle microtubules. $\mathrm{Hecl}$ is phosphorylated by $\mathrm{Au}-$ rora B kinase on as many as nine target sites within its unstructured "tail" domain to tune the affinity of kinetochores for microtubules throughout mitosis (Cheeseman et al. 2006; DeLuca et al. 2006, 2011; Alushin et al. 2012; Zaytsev et al. 2014, 2015). High levels of phosphorylation promote kinetochore-microtubule turnover, which facilitates attachment error correction in early mitosis, whereas low levels of Hec1 phosphorylation result in stabilized attachments in late mitosis. Hec1 is arguably a main effector of Aurora B kinase-mediated regulation of attach- ment stability, however additional kinetochore proteins (e.g., Dsn1, Knl1, Ska1, Ska3, and CENP-E) are phosphorylated by Aurora B, which likely contributes to kinetochore-microtubule attachment regulation (Kim et al. 2010; Welburn et al. 2010; Chan et al. 2012).

Although Aurora B is well-established as the "master regulator" of kinetochore-microtubule attachment stability, there is growing evidence that it does not work alone to phosphorylate kinetochore substrates to promote microtubule turnover. A number of recent studies suggest that Aurora A kinase, which has well-known functions at spindle poles in mitosis, additionally plays a role at the kinetochore-microtubule interface to ensure proper regulation of attachments during mitotic progression. How the division of labor between Aurora A and Aurora B is coordinated to ensure proper spatial and temporal control of kinetochore-microtubule attachment stability is an important question that has gained attention in recent years.

\section{THE AURORA KINASE FAMILY}

The Aurora kinases are a highly conserved family of serine/threonine kinases that have essential functions in organisms across evolution. Budding and fission yeast have just one Aurora kinase (Ipl1 and Ark1, respectively), whereas mammals typically have three family members: Aurora A, B, and C (Chan and Botstein 1993; Bischoff and Plowman 1999; Giet and Prigent 1999; Adams et al. 2001; Petersen et al. 2001). In humans, Aurora A and Aurora B have essential functions in mitotic cell division (Bolanos-Garcia 2005; Barr and Gergely 2007; Vader and Lens 2008; Krenn and Musacchio 2015). The third human family member, Aurora C, plays a role in meiotic cell division, as well as the first mitotic divisions of the mammalian zygote (Fernandez-Miranda et al. 2011; Yang et al.

(C) 2017 DeLuca. This article is distributed under the terms of the Creative Commons Attribution-NonCommercial License, which permits reuse and redistribution, except for commercial purposes, provided that the original author and source are credited. 
2015; Nguyen and Schindler 2017). The Aurora kinases share $\sim 70 \%$ homology in their catalytic domains, although their localization patterns and substrates are distinct, resulting in unique roles during cell division (Carmena et al. 2009; Nguyen and Schindler 2017).

\section{Aurora A Kinase}

Aurora A localizes primarily to spindle poles throughout mitosis and has roles in centrosome maturation, centrosome separation, and bipolar spindle assembly (Katayama et al. 2003; Crane et al. 2004; Ducat and Zheng 2004; Barr and Gergely 2007). Activation of Aurora A is promoted by interaction with its well-characterized cofactor TPX2, a microtubule binding protein that localizes to spindle poles and microtubules (Kufer et al. 2002; Bayliss et al. 2003; Eyers and Maller 2003, 2004; Ozlu et al. 2005). Although TPX2 is considered the predominant cofactor for Aurora A, other proteins also participate in its activation including Ajuba, Bora, Pak2, and Inhibitor-2 (Hirota et al. 2003; Satinover et al. 2004; Zhao et al. 2005; Hutterer et al. 2006; Seki et al. 2008; Carmena et al. 2009). The ability of Aurora A to carry out its functions in centrosome separation and spindle assembly is facilitated through its targeting to these locations by binding partners (e.g., to spindle microtubules by TPX2; to centrosomes by Cep192) (Kufer et al. 2002; Glover 2003; Joukov et al. 2010; Bertolin et al. 2016). Although the majority of studies regarding Aurora A have focused on its functions in centrosome separation and spindle assembly, there is growing evidence that it has additional roles in facilitating successful chromosome alignment and segregation, which will be the focus of this review.

\section{Aurora B Kinase}

Aurora B localizes to the centromere/kinetochore region of mitotic chromosomes from late prophase to metaphase and then relocates to the spindle midzone at anaphase onset (Earnshaw and Bernat 1991; Bischoff and Plowman 1999; Giet and Prigent 1999). All functions of Aurora B studied to date require its incorporation into the chromosome passenger complex (CPC), which additionally contains INCENP, Survivin, and Borealin (Adams et al. 2001; Wheatley et al. 2001; Bolton et al. 2002; Honda et al. 2003; Gassmann et al. 2004; Vader et al. 2006). The carboxyl terminus of INCENP binds to and increases the catalytic activity of Aurora B, whereas Survivin, Borealin, and the amino terminus of INCENP form a module that facilitates localization of the entire CPC to centromeres in early mitosis and to the spindle midzone in late mitosis (Bishop and Schumacher 2002; Honda et al. 2003; Yasui et al. 2004; Sessa et al. 2005; Klein et al. 2006; Vader et al. 2006; Jeyaprakash et al. 2007). In early mitosis, Aurora B has roles in kinetochore assembly, activation of the spindle assembly checkpoint, and kinetochore-microtubule error correction (Biggins et al. 1999; Kallio et al. 2002; MurataHori et al. 2002; Murata-Hori and Wang 2002; Tanaka et al. 2002; Carvalho et al. 2003; Hauf et al. 2003; Lens et al. 2003; Liu et al. 2006; Santaguida et al. 2011). To execute its error correction function, Aurora B phosphorylates kinetochore substrates, including the microtubule attachment factor $\mathrm{Hec} 1$, to promote kinetochore-microtubule turnover (Cheeseman et al. 2006; DeLuca et al. 2006; Welburn et al. 2010). Rapid turnover of kinetochore microtubules in early mitosis, facilitated through high levels of Hecl phosphorylation, prevents the accumulation of erroneous kinetochore-microtubule attachments during mitotic progression. Although Aurora B kinase-mediated phosphorylation of Hec1 markedly decreases by metaphase, some level of phosphorylation of $\mathrm{Hec} 1$ is required in this later stage of mitosis for kinetochores to fluidly track the growing and shortening ends of attached microtubules (DeLuca et al. 2011; Zaytsev et al. 2014). At anaphase onset, Aurora B relocalizes to the spindle midzone where it functions in stabilization of the central spindle and regulation of cytokinesis (Cooke et al. 1987; Eckley et al. 1997; Schumacher et al. 1998; Tatsuka et al. 1998; Terada et al. 1998; Bischoff and Plowman 1999; Adams et al. 2000; Kaitna et al. 2000; Giet and Glover 2001). For further discussion of Aurora B kinase and its mitotic functions, the reader is directed to recent reviews (Carmena et al. 2012; van der Horst and Lens 2014; Krenn and Musacchio 2015; Afonso et al. 2017).

\section{Aurora C Kinase}

Aurora $\mathrm{C}$ is primarily expressed in germ cells and participates in the regulation of chromosome-microtubule interactions during meiosis and mitotic divisions of the early embryo (Tang et al. 2006; Sharif et al. 2010; Yang et al. 2010; Fernandez-Miranda et al. 2011; Schindler et al. 2012; Balboula and Schindler 2014). Meiotic oocytes contain two forms of the CPC: one containing Aurora B and one containing Aurora C. Based on early studies demonstrating that Aurora $\mathrm{C}$ could compensate for mitotic Aurora B functions, Aurora C was presumed to be the meiotic homolog of Aurora B (Sasai et al. 2004). However, recent studies have suggested nonredundant functions of the kinases and also showed that the two kinases localize to distinct structures in meiotic cells. Aurora B predominantly resides on kinetochores, where it contributes to chromosome alignment, and Aurora C localizes to spindle poles, centromeres, and the interchromatid axis, where it is reported to have roles in spindle assembly and in correction of kinetochore-microtubule attachment errors (Shuda et al. 2009; Balboula and Schindler 2014; Nguyen et al. 2014; Fellmeth et al. 2015; Balboula et al. 2016; Sasai et al. 2016; Quartuccio et al. 2017). It is also noteworthy that although Aurora $\mathrm{C}$ is primarily expressed in germ line cells, it is also expressed in human tumor cells, and its overexpression in noncancerous cells can result in cell transformation (Ehara et al. 2003; Dutertre et al. 2005; Ulisse et al. 2006; Khan et al. 2011; Tsou et al. 2011). Discussions of Aurora $\mathrm{C}$ kinase and its functions can be found in recent reviews (Yang et al. 2015; Nguyen and Schindler 2017). 


\section{KINETOCHORE FUNCTIONS OF AURORA A KINASE}

A little over 15 years ago, two studies reported a role for Aurora A kinase in chromosome alignment, independent of its role in spindle pole separation. Kunitoku et al. (2003) found that a small population of human cells depleted of Aurora A by RNAi managed to enter mitosis and form bipolar spindles. These cells experienced defects in chromosome alignment, with most mitotic cells exhibiting pole-localized chromosomes. Similarly, Marumoto et al. (2003) reported chromosome alignment defects after microinjection of Aurora A antibodies into prometaphase human cells with separated spindle poles. In the former study, the authors identified the kinetochore protein CENP-A as an Aurora A binding partner through a yeast two-hybrid approach and mapped Ser7 as a bona fide Aurora A substrate whose phosphorylation in prophase is dependent on Aurora A activity. The authors went on to show that expression of a nonphosphorylatable CENP-A (Ser7 mutated to Alanine) also resulted in chromosome misalignment. Because CENP-A Ser7 is also a target of Aurora B (Zeitlin et al. 2001), it remains unclear whether the observed defects in cells expressing a nonphosphorylatable CENP-A Ser7 mutant are related to Aurora A or to Aurora B-mediated phosphorylation. Still, these studies uncovered a potential new role for Aurora A in mitotic chromosome alignment and segregation beyond mitotic entry and spindle pole separation. More recently, additional Aurora A kinase substrates have been identified at the kinetochore, and their phosphorylation specifically by Aurora A has been shown to be essential for regulation of kinetochore-microtubule attachments. Work from multiple laboratories has demonstrated that there are at least two mechanisms for Aurora A-mediated phosphorylation of kinetochore substrates. In the first, spindle pole-localized Aurora A phosphorylates kinetochores on chromosomes that occupy a position, even transiently, near one of the two spindle poles (Kim et al. 2010; Chmátal et al. 2015; Ye et al. 2015). In the second, Aurora A is recruited to the kinetochore region to phosphorylate substrates there, independent of chromosome proximity to a spindle pole (DeLuca et al. 2018). Both mechanisms likely play an important role in temporally regulating kinetochoremicrotubules during mitosis, and are discussed in detail below.

\section{Kinetochore Substrate Phosphorylation by Pole-Associated Aurora A}

The kinetochore-associated, plus end-directed microtubule motor protein CENP-E transports chromosomes along spindle microtubules from spindle poles to the metaphase plate in mitosis (Kapoor et al. 2006). In 2010, Kim et al. demonstrated that CENP-E Thr422 is targeted by both Aurora A and Aurora B kinases, and phosphorylation at this site decreases CENP-E-mediated recruitment of PP1 (a phosphatase that promotes attachment stability by counteracting Aurora B kinase activity) and reduces affinity of CENP-E for microtubules. The authors found that centrosome-proximal chromosomes exhibited high levels of Thr422 phosphorylation, presumably due to active Aurora A at spindle poles and active Aurora B at the centromere/kinetochore region. In this scenario, the authors reasoned that incorrect attachments near spindle poles (i.e., syntelic attachments, in which both sister kinetochores are attached to microtubules from a single spindle pole) become destabilized, allowing for subsequent formation of correct attachments and productive CENP-E-powered movements of chromosomes toward the spindle equator (Kim et al. 2010). These findings suggest a role for spindle pole-associated Aurora A kinase in phosphorylating kinetochore substrates to promote correction of attachment errors specifically on chromosomes that become "trapped" near the centrosome region.

Hec1 was also recently identified as a kinetochore substrate of spindle-pole associated Aurora A kinase both in mitotic and meiotic cells. In a study by Ye et al., the authors found that in Drosophila S2 cells, overexpression of Aurora A resulted in enrichment of the kinase at spindle poles and a reduced incidence of experimentally induced syntelic attachments (Ye et al. 2015). In a reciprocal experiment, RNAi-mediated depletion of Aurora A resulted in increased pole-associated, syntelically attached kinetochores. The authors expanded their study into PtK1 cells, and reported a significant increase in pole-oriented chromosomes in cells treated with an Aurora A-specific kinase inhibitor (Ye et al. 2015). They went on to confirm that Hec1 Ser55 is not only an Aurora B target site, but also a substrate of Aurora A whose phosphorylation decreased in cells treated with Aurora A inhibitors (DeLuca et al. 2011; Kettenbach et al. 2011; Ye et al. 2015). In a companion study, Chmátal et al. (2015) demonstrated a similar role for spindle pole-associated Aurora A kinase in dividing meiotic cells. The authors found that during meiosis I in mouse oocytes, microtubule attachments to kinetochores on pole-proximal chromosomes were specifically destabilized, and this effect was reduced upon inhibition of Aurora A kinase (Chmátal et al. 2015). Combined with the observation that Aurora B kinase activity is high on pole-proximal chromosomes and contributes to the destabilization of kinetochore-microtubule attachments on these chromosomes (Maia et al. 2010), these studies suggest that $\mathrm{Hec} 1$ phosphorylation by both Aurora B kinase and pole-associated Aurora A kinase plays an important role in the correction of syntelic attachments in early mitotic cells.

It is well-established that Aurora B phosphorylates substrates at the centromere/kinetochore region. From recent work it has become clear that spindle pole-associated Aurora A also phosphorylates kinetochore substrates, and in fact, the two kinases may act together on the same substrates. This leads to a model whereby the degree of Aurora-mediated phosphorylation on a particular substrate is dictated by the landscape of chromosome distribution within a mitotic cell. This model reinforces the dogma that Aurora A and Aurora B are specifically compartmentalized within mitotic cells and that phosphorylation depends on substrate localization to these compartments. 


\section{Kinetochore Substrate Phosphorylation by Centromere/Kinetochore-Associated Aurora A}

In a recent study, we found that Hec1 Ser69, which was previously identified as an in vitro substrate of Aurora B kinase (DeLuca et al. 2006), is primarily phosphorylated by Aurora A kinase in both human and PtK1 cells. Inhibition of Aurora A, but not Aurora B kinase, resulted in markedly reduced levels of phosphorylation at this site on kinetochores. Unlike the other characterized Hec1 tail domain target sites, phosphorylation of Ser69 persists at high levels throughout the entirety of mitosis (DeLuca et al. 2018). Furthermore, we found that Aurora A-mediated phosphorylation of Ser69 is required for normal kinetochore oscillatory movements at metaphase. Given the well-established localization of Aurora A at spindle poles, persistent phosphorylation of an Aurora A-specific kinetochore substrate in late mitosis was surprising, because kinetochores are maximally distant from poles in metaphase. We first hypothesized, consistent with the compartmentalization model described above, that Aurora A phosphorylates Ser69 in early mitosis when chromosomes are likely to encounter a spindle pole, and this phosphorylation persists throughout mitosis. However, when we allowed mitotic cells to progress to metaphase and added Aurora A inhibitors only after chromosomes had completely aligned, immunofluorescence experiments revealed a near-complete loss of both Ser69 phosphorylation and kinetochore oscillations (DeLuca et al. 2018). These studies demonstrate that Aurora A kinase is able to phosphorylate kinetochores on chromosomes distal from the spindle poles, and that this activity is required for regulation of kinetochore-microtubule attachments. As such, they compel a re-evaluation of the Aurora kinase compartmentalization model and beg the question of how Aurora A is recruited to kinetochores in metaphase.

\section{AURORA A RECRUITMENT TO THE CENTROMERE/KINETOCHORE REGION}

It is relatively easy to envision a scenario in which Aurora A kinase is spatially restricted in a mitotic cell, and its phosphorylation of kinetochore substrates is dictated by chromosome location. In this case, the kinase resides at spindle poles and phosphorylates kinetochores only on chromosomes that migrate within range of the localized kinase. Aurora A would be ideally positioned to reduce the incidence of chromosomes that are trapped at spindle poles through both prevention and correction of erroneous kinetochore-microtubule attachments, a phenomenon reported in previous studies (Chmátal et al. 2015; Ye et al. 2015). However, it has become clear that Aurora A also phosphorylates kinetochore substrates on chromosomes that are aligned at the spindle equator, distant from spindle poles (DeLuca et al. 2018). It is more difficult to envision a scenario in which a spindle polerestricted kinase is able to phosphorylate distal kinetochore substrates. Several possibilities for how this might be achieved are discussed below.

In the first, Aurora A is directly recruited to the centromere/kinetochore region via INCENP (in complex with the other CPC components). In this model, a population of Aurora A is bound to INCENP rather than TPX2. In support of this model, the Sen laboratory demonstrated through immunoprecipitation experiments that Aurora A forms distinct complexes with both TPX2 and INCENP during mitosis and that Aurora A directly interacts with INCENP in vitro (Katayama et al. 2008). In contrast, Aurora B kinase interacted only with INCENP, and not TPX2 (Katayama et al. 2008). In this study, the authors carried out a time-course immunoprecipitation experiment to determine the timing of Aurora A and Aurora B complex formation with INCENP. Interestingly, Aurora B/INCENP was detectable over a broader timescale during mitosis than Aurora A/INCENP, which was evident during only a short window during mitosis, and whose abundance peaked after Aurora B/INCENP (Katayama et al. 2008). This suggests that Aurora A may only bind INCENP in late mitosis, lending support to the idea that the kinase might switch from binding TPX2 to INCENP, allowing for recruitment to the centromere region specifically in metaphase. Our recent results confirm that Aurora A associates with INCENP during mitosis, and specifically that the interaction of INCENP with Aurora A is mediated through INCENP's IN-box domain, the domain required for Aurora B binding (Fu et al. 2009; DeLuca et al. 2018). Furthermore, depletion of INCENP by RNAi in human cells significantly reduced phosphorylation of $\mathrm{Hecl}$ Ser69. Because this site is primarily phosphorylated by Aurora A in cells, this result suggests that an INCENPAurora A kinase complex may indeed phosphorylate Hec1 (DeLuca et al. 2018). It will be important in future studies to determine how a potential switch between TPX2- and INCENP-Aurora A association is regulated in cells to ensure a timely hand-off between the two regulators.

A second possibility is that Aurora A is directly recruited to outer kinetochores, independent of INCENP, to phosphorylate kinetochore proteins. Chmátal et al. found that Aurora A kinase localizes to kinetochores in mouse meiotic cell division, suggesting kinetochores have the inherent ability to bind Aurora A (Chmátal et al. 2015). In addition, Hans et al. showed that a truncated version of Aurora A kinase missing its amino-terminal 120 amino acids localizes readily to kinetochores in human mitotic cells depleted of endogenous Aurora B (Hans et al. 2009). Strikingly, this localization was not dependent on the ability of Aurora A to bind INCENP, and furthermore, the kinetochore-associated, truncated Aurora A kinase did not exhibit "passenger"-like activity at anaphase onset, but instead remained bound to kinetochores (Hans et al. 2009). These studies raise the interesting possibility that Aurora A may be temporally regulated, such that in early mitosis, it is largely spindle pole-associated, and in late mitosis a population is modified such that it directly binds kinetochores. However, this may be unlikely, because Aurora $\mathrm{A}$ is not readily detectable at kinetochores in unperturbed mitotic cells.

A third possibility is that kinetochore substrates are phosphorylated by a population of Aurora A kinase that is delivered to the vicinity of kinetochores by spindle microtubules. Both Aurora A and its activator TPX2 associ- 
ate with spindle microtubules, and in fact, an additional cofactor, RHAMM (a TPX2 and microtubule binding protein) has been identified both on spindle microtubules and kinetochores (Chen et al. 2014). Finally, in a fourth possibility, it is feasible that Aurora A does not require recruitment, per se, to kinetochores, but instead a population of soluble, cytoplasmic kinase is sufficient to phosphorylate kinetochores during late mitosis. A recent study demonstrated such a phenomenon for Aurora B. In this case, an experimentally activated version of the kinase was mutated such that it could not bind the centromere/ kinetochore region; however, it maintained the ability to phosphorylate outer kinetochore substrates during mitosis (Haase et al. 2017).

Phosphorylation of kinetochore substrates by Aurora A kinase occurs when chromosomes are near spindle poles (in prometaphase) and distal from spindle poles (in metaphase), and both activities contribute to accurate, errorfree chromosome segregation. The mechanism by which Aurora A phosphorylates kinetochores in early mitosis on pole-associated chromosomes is likely straightforward, given the high levels of Aurora A at spindle poles. In contrast, it has been difficult to detect endogenous Aurora A at kinetochores or centromeres during mitosis, therefore the mechanism by which the kinase phosphorylates kinetochore substrates in metaphase remains unresolved. Future studies using approaches to detect transient proteinprotein interactions at kinetochores should help further our understanding of these mechanisms.

\section{AURORA KINASE SUBSTRATE SPECIFICITY}

Consistent with the ability of Aurora A and B to phosphorylate the same substrates in vitro (Vader and Lens 2008; Carmena et al. 2009), the consensus sequences recognized by the two kinases are quite similar, with only subtle distinctions (Meraldi et al. 2004; Ohashi et al. 2006; Kim et al. 2010; Kettenbach et al. 2011; Koch et al. 2011). Given comparable consensus sequences and no compelling evidence indicating substrate preference in vitro, how is specificity achieved in cells? As discussed above, the prevailing model is that Aurora kinases are recruited to specific subcellular localizations to phosphorylate targets at these sites. This compartmentalization model predicts that targeting either kinase to the other kinase's endogenous location should result in functional replacementmeaning that each kinase should be able to phosphorylate the other kinase's substrates, as long as it is targeted to the appropriate location in the cell. This was tested by two groups in 2009. In both studies, the authors demonstrated that mutation of a single amino acid in Aurora A, Gly198, to the analogous residue in Aurora B, an Asparagine, resulted in loss of Aurora A localization to spindle poles, and subsequent relocalization to centromeres prior to anaphase and to the spindle midzone after anaphase onset. Strikingly, in Aurora B-depleted cells, this mutant version of Aurora A was able to functionally replace Aurora B, and cells progressed through mitosis without detectable chromosome segregation defects (Fu et al. 2009; Hans et al.
2009). Furthermore, in vitro studies revealed that this single mutation increased Aurora A's ability to bind INCENP and Survivin (Fu et al. 2009; Hans et al. 2009). The authors concluded that the activity of Aurora A and Aurora B toward spindle pole and centromere/kinetochore substrates, respectively, in unperturbed mitotic cells is a consequence of spatial compartmentalization. In further support of this idea, a recent study showed that ectopically targeting Aurora A to either mitotic chromatin (fusion to Histone $\mathrm{H} 2 \mathrm{~B}$ ), centromeres (fusion to CENP-B), or kinetochores (fusion to $\mathrm{Hec} 1$ ) resulted in rescue of Aurora B function at each of those locations in cells depleted of endogenous Aurora B (Li et al. 2015). Conversely, ectopically targeting Aurora B to spindle poles (fusion to a fragment of centrosome component PLK4), rescued the function of Aurora A kinase at spindle poles in cells depleted of Aurora A (Li et al. 2015). Together, these studies support a model in which the specificity of the Aurora kinases for their substrates is dictated in large part by location within the cell.

Our recent work, however, suggests that this model may not entirely explain why certain substrates are phosphorylated preferentially by one Aurora kinase over the other during mitosis. We found that Aurora A and Aurora B contribute to phosphorylation of Hec1 tail domain target sites (of the three tested) to varying degrees in mammalian cells. In early mitosis, Aurora A kinase robustly phosphorylates Hec1 Ser69 and Ser55, but only minimally contributes to phosphorylation of closely neighboring Ser44 (DeLuca et al. 2018). Thus, in early mitosis, when Hec1 tail domain substrates are similarly exposed to both Aurora A and Aurora B, the kinases exhibit preference for certain sites, indicating that spatial compartmentalization is not the only determinant of kinase specificity in cells. Inherent bias for one site over another likely does not explain this phenomenon, because we found that in vitro, all sites are phosphorylated by both kinases, with little preference for any individual site(s) (DeLuca et al. 2018). We cannot rule out the possibility, however, that our in vitro assay could not resolve such preferences. Perhaps even more intriguing, during metaphase, we observed a marked reduction in Ser55 phosphorylation, whereas Ser69 phosphorylation remained high, and this sustained phosphorylation was dependent on Aurora A activity. At this point in mitosis, Aurora B activity is low at kinetochores, and our data demonstrating that Ser44 phosphorylation levels are maximally low at metaphase support this premise (DeLuca et al. 2011). These results suggest that the specificity of Aurora A kinase toward distinct kinetochore target sites may be differentially regulated. We tested to see if the position of a particular target site within the $\mathrm{Hec} 1$ tail may give rise to this specificity (i.e., Ser69 may be "accessible" to Aurora A, whereas Ser55 may not). For this experiment, we generated a tail "domain swap" mutant in which regions of the Hec1 tail were transposed, resulting in Ser55 occupying a position near the Hec1 globular domain (similar to Ser69's position in the wild-type tail), and Ser69 occupying a position toward the aminoterminal, distal region of the tail. The temporal phosphorylation patterns of Ser55 and Ser69 during mitotic progression were identical in wild-type and mutant $\mathrm{Hec} 1$, and 
the sensitivity of phosphorylation at each site to Aurora A and Aurora B inhibitors was also unchanged (DeLuca et al. 2018), leading us to conclude that site specificity is not dictated by spatial positioning within the tail. How then is specificity achieved? Although this remains an important area for future investigation, an interesting possibility is that phosphorylation on individual Hec1 sites varies as a consequence of differential dephosphorylation. The kinetochore phosphatases responsible for dephosphorylation of the Hec1 target sites remain unknown, but their identification and functional characterization will no doubt aid in understanding how different phosphorylation states of individual Aurora substrates are achieved in mitosis.

\section{WHY DO CELLS REQUIRE AURORA A- MEDIATED KINETOCHORE REGULATION?}

These studies raise the question of why mammalian cells require Aurora A, in addition to Aurora B, to facilitate kinetochore-microtubule attachment regulation? In early mitosis, it is important that global kinetochore-microtubule attachment turnover is high to prevent premature stabilization of kinetochore-microtubules. It is well-established that Aurora B kinase, which is highly concentrated at the centromere/kinetochore region in early mitosis, contributes to this error-correction (and error-prevention) mechanism. During prometaphase, mono-oriented chromosomes are typically transported to spindle poles by the minus end-directed microtubule motor protein dynein to promote chromosome alignment and biorientation. However, while localized near a spindle pole, chromosomes risk forming syntelic attachments. Thus, it is important that cells have a robust mechanism to ensure that these erroneous attachments are corrected (or avoided altogether) on pole-localized chromosomes. Aurora A kinase at spindle poles helps solve this problem and works together with Aurora B (Maia et al. 2010; Krenn and Musacchio 2015) to ensure that kinetochore substrates such as Hec1 are highly phosphorylated to promote kinetochore-microtubule turnover.

The role for Aurora A kinase in metaphase appears to be distinct from its role in early mitosis. As discussed above, mutation of the Aurora A substrate Hec1 Ser69 to Alanine or cell-wide inhibition of Aurora A kinase activity results in markedly decreased kinetochore oscillations. These perturbations result in a small but significant increase in chromosome segregation errors - about a twofold increase in lagging chromosomes in anaphase in human cells (DeLuca et al. 2018). A likely cause for these errors is a failure to correct merotelic attachments (i.e., one kinetochore of a sister pair is bound to microtubules emanating from both poles), which is known to increase the incidence of lagging chromosomes in anaphase (Cimini et al. 2001). In support of this notion, Ye et al. (2015) found in PtK1 cells that inhibition of Aurora A kinase activity resulted in a 3.6-fold increase in merotelic attachments at metaphase, and a corresponding 3.2-fold increase in lagging chromosomes in anaphase. Together, these studies suggest a role for Aurora A kinase in regu- lating kinetochore-microtubule dynamics in late mitosis. These results also support the idea that kinetochore oscillations in late mitosis contribute to mitotic fidelity. This is noteworthy, because the role for oscillations in mitosis has yet to be resolved. One possibility is that oscillations provide increased kinetochore-microtubule turnover to detach any merotelic attachments that may remain in metaphase (Cimini et al. 2003). However, this mechanism may not be universally conserved, because there are cell types that do not exhibit metaphase oscillations (e.g., embryonic cells of Drosophila and Xenopus) (Desai et al. 1998; Maddox et al. 2002) but have no obvious increased incidence of lagging chromosomes. Interestingly, spindles in these cells have high rates of flux (i.e., the continual addition of tubulin dimers at microtubule plus ends and a corresponding loss from microtubule minus ends at spindle poles), which may provide the high turnover of tubulin subunits at the kinetochore-microtubule interface to promote release of erroneous attachments (Desai et al. 1998; BrustMascher and Scholey 2002; Maddox et al. 2002, 2003). Although the physiological reason for kinetochore oscillations remains an important topic to be explored, it is clear that Aurora A kinase is required for late mitotic kinetochore-microtubule attachment regulation to ensure error-free chromosome segregation.

\section{CONCLUSION}

Precise regulation of kinetochore-microtubule attachment stability is essential for ensuring the fidelity of chromosome segregation during mitosis. Research over the last several decades has established that Aurora B kinase is the master controller of this process, however, recent studies have revealed that its sister kinase, Aurora A, likely plays a supporting role in this regulation. From these studies, a model emerges in which Aurora A, concentrated at mitotic spindle poles, phosphorylates kinetochore substrates on pole-proximal chromosomes (together with centromere/ kinetochore-localized Aurora B) to ensure both the prevention and correction of attachment errors in early mitosis. As chromosomes align and Aurora B kinase activity decreases at kinetochores, Aurora A continues to phosphorylate outer kinetochore substrates which permits the fluid tracking of dynamic microtubule plus-ends and chromosome oscillatory movements. Many questions regarding this duel regulation still remain: How is Aurora A recruited to the centromere/kinetochore region? What determines site specificity for Aurora A versus Aurora B in cells? Are unique phosphatase complexes used to dephosphorylate different Aurora-mediated phosphorylation events? Addressing these questions will be critical in unraveling how the Aurora kinases ensure correct and timely chromosome segregation during mitotic cell division.

\section{ACKNOWLEDGMENTS}

This work is supported by grant R01GM088371 from the National Institutes of Health. I thank Keith DeLuca and Dr. Susanne Lens for critical comments on the manuscript. 


\section{REFERENCES}

Adams RR, Wheatley SP, Gouldsworthy AM, Kandels-Lewis SE, Carmena M, Smythe C, Gerloff DL, Earnshaw WC. 2000. INCENP binds the Aurora-related kinase AIRK2 and is required to target it to chromosomes, the central spindle and cleavage furrow. Curr Biol 10: 1075-1078.

Adams RR, Carmena M, Earnshaw WC. 2001. Chromosomal passengers and the (aurora) ABCs of mitosis. Trends Cell Biol 11: 49-54.

Afonso O, Figueiredo AC, Maiato H. 2017. Late mitotic functions of Aurora kinases. Chromosoma 126: 93-103.

Alushin GM, Musinipally V, Matson D, Tooley J, Stukenberg PT, Nogales E. 2012. Multimodal microtubule binding by the Ndc80 kinetochore complex. Nat Struct Mol Biol 19: 11611167.

Balboula AZ, Schindler K. 2014. Selective disruption of aurora C kinase reveals distinct functions from aurora B kinase during meiosis in mouse oocytes. PLoS Genet 10: e1004194.

Balboula AZ, Nguyen AL, Gentilello AS, Quartuccio SM, Drutovic D, Solc P, Schindler K. 2016. Haspin kinase regulates microtubule-organizing center clustering and stability through Aurora kinase C in mouse oocytes. J Cell Sci 129: 3648-3660.

Barr AR, Gergely F. 2007. Aurora-A: The maker and breaker of spindle poles. J Cell Sci 120: 2987-2996.

Bayliss R, Sardon T, Vernos I, Conti E. 2003. Structural basis of Aurora-A activation by TPX2 at the mitotic spindle. Mol Cell 12: 851-862.

Bertolin G, Sizaire F, Herbomel G, Reboutier D, Prigent C, Tramier M. 2016. A FRET biosensor reveals spatiotemporal activation and functions of aurora kinase A in living cells. Nat Commun 7: 12674.

Biggins S, Severin FF, Bhalla N, Sassoon I, Hyman AA, Murray AW. 1999. The conserved protein kinase Ipl1 regulates microtubule binding to kinetochores in budding yeast. Genes Dev 13: $532-544$.

Bischoff JR, Plowman GD. 1999. The Aurora/Ipllp kinase family: Regulators of chromosome segregation and cytokinesis. Trends Cell Biol 9: 454-459.

Bishop JD, Schumacher JM. 2002. Phosphorylation of the carboxyl terminus of inner centromere protein (INCENP) by the Aurora B Kinase stimulates Aurora B kinase activity. $J$ Biol Chem 277: 27577-27580.

Bolanos-Garcia VM. 2005. Aurora kinases. Int J Biochem Cell Biol 37: 1572-1577.

Bolton MA, Lan W, Powers SE, McCleland ML, Kuang J, Stukenberg PT. 2002. Aurora B kinase exists in a complex with survivin and INCENP and its kinase activity is stimulated by survivin binding and phosphorylation. Mol Biol Cell 13: 3064-3077.

Brust-Mascher I, Scholey JM. 2002. Microtubule flux and sliding in mitotic spindles of Drosophila embryos. Mol Biol Cell 13: 3967-3975.

Carmena M, Ruchaud S, Earnshaw WC. 2009. Making the Auroras glow: Regulation of Aurora A and B kinase function by interacting proteins. Curr Opin Cell Biol 21: 796-805.

Carmena M, Wheelock M, Funabiki H, Earnshaw WC. 2012. The chromosomal passenger complex (CPC): From easy rider to the godfather of mitosis. Nat Rev Mol Cell Biol 13: 789803

Carvalho A, Carmena M, Sambade C, Earnshaw WC, Wheatley SP. 2003. Survivin is required for stable checkpoint activation in taxol-treated HeLa cells. J Cell Sci 116: 2987-2998.

Chan CS, Botstein D. 1993. Isolation and characterization of chromosome-gain and increase-in-ploidy mutants in yeast. Genetics 135: 677-691.

Chan YW, Jeyaprakash AA, Nigg EA, Santamaria A. 2012. Aurora $\mathrm{B}$ controls kinetochore-microtubule attachments by inhibiting Ska complex-KMN network interaction. J Cell Biol 196: 563-571
Cheeseman IM, Chappie JS, Wilson-Kubalek EM, Desai A. 2006. The conserved KMN network constitutes the core microtubule-binding site of the kinetochore. Cell 127: 983-997.

Chen H, Mohan P, Jiang J, Nemirovsky O, He D, Fleisch MC, Niederacher D, Pilarski LM, Lim CJ, Maxwell CA. 2014. Spatial regulation of Aurora A activity during mitotic spindle assembly requires RHAMM to correctly localize TPX2. Cell Cycle 13: 2248-2261.

Chmátal L, Yang K, Schultz RM, Lampson MA. 2015. Spatial regulation of kinetochore microtubule attachments by destabilization at spindle poles in meiosis I. Curr Biol 25: 1835-1841.

Cimini D, Howell B, Maddox P, Khodjakov A, Degrassi F, Salmon ED. 2001. Merotelic kinetochore orientation is a major mechanism of aneuploidy in mitotic mammalian tissue cells. J Cell Biol 153: 517-527.

Cimini D, Moree B, Canman JC, Salmon ED. 2003. Merotelic kinetochore orientation occurs frequently during early mitosis in mammalian tissue cells and error correction is achieved by two different mechanisms. J Cell Sci 116: 4213-4225.

Cooke CA, Heck MM, Earnshaw WC. 1987. The inner centromere protein (INCENP) antigens: Movement from inner centromere to midbody during mitosis. J Cell Biol 105: 20532067.

Crane R, Gadea B, Littlepage L, Wu H, Ruderman JV. 2004. Aurora A, meiosis and mitosis. Biol Cell 96: 215-229.

DeLuca JG, Gall WE, Ciferri C, Cimini D, Musacchio A, Salmon ED. 2006. Kinetochore microtubule dynamics and attachment stability are regulated by Hec1. Cell 127: 969-982.

DeLuca KF, Lens SM, DeLuca JG. 2011. Temporal changes in Hec1 phosphorylation control kinetochore-microtubule attachment stability during mitosis. J Cell Sci 124: 622-634.

DeLuca KF, Meppelink A, Broad AJ, Mick JE, Peersen OB, Pektas S, Lens SMA, DeLuca JG. 2018. Aurora A kinase phosphorylates Hec1 to regulate metaphase kinetochore-microtubule dynamics. J Cell Biol 217: 163-177.

Desai A, Maddox PS, Mitchison TJ, Salmon ED. 1998. Anaphase A chromosome movement and poleward spindle microtubule flux occur at similar rates in Xenopus extract spindles. $J$ Cell Biol 141: 703-713.

Ducat D, Zheng Y. 2004. Aurora kinases in spindle assembly and chromosome segregation. Exp Cell Res 301: 60-67.

Dutertre S, Hamard-Peron E, Cremet JY, Thomas Y, Prigent C. 2005. The absence of p53 aggravates polyploidy and centrosome number abnormality induced by Aurora- $\mathrm{C}$ overexpression. Cell Cycle 4: 1783-1787.

Earnshaw WC, Bernat RL. 1991. Chromosomal passengers: Toward an integrated view of mitosis. Chromosoma 100: 139146.

Eckley DM, Ainsztein AM, Mackay AM, Goldberg IG, Earnshaw WC. 1997. Chromosomal proteins and cytokinesis: Patterns of cleavage furrow formation and inner centromere protein positioning in mitotic heterokaryons and mid-anaphase cells. J Cell Biol 136: 1169-1183.

Ehara H, Yokoi S, Tamaki M, Nishino Y, Takahashi Y, Deguchi T, Kimura M, Yoshioka T, Okano Y. 2003. Expression of mitotic Aurora/Ipl1p-related kinases in renal cell carcinomas: An immunohistochemical study. Urol Res 31: 382-386.

Eyers PA, Maller JL. 2003. Regulating the regulators: Aurora A activation and mitosis. Cell Cycle 2: 287-289.

Eyers PA, Maller JL. 2004. Regulation of Xenopus Aurora A activation by TPX2. J Biol Chem 279: 9008-9015.

Fellmeth JE, Gordon D, Robins CE, Scott RT Jr, Treff NR, Schindler K. 2015. Expression and characterization of three Aurora kinase C splice variants found in human oocytes. Mol Hum Reprod 21: 633-644.

Fernandez-Miranda G, Trakala M, Martin J, Escobar B, Gonzalez A, Ghyselinck NB, Ortega S, Canamero M, Perez de Castro I, Malumbres M. 2011. Genetic disruption of aurora B uncovers an essential role for aurora $\mathrm{C}$ during early mammalian development. Development 138: 2661-2672.

Fu J, Bian M, Liu J, Jiang Q, Zhang C. 2009. A single amino acid change converts Aurora-A into Aurora-B-like kinase in terms 
of partner specificity and cellular function. Proc Natl Acad Sci 106: 6939-6944.

Funabiki H, Wynne DJ. 2013. Making an effective switch at the kinetochore by phosphorylation and dephosphorylation. Chromosoma 122: 135-158.

Gassmann R, Carvalho A, Henzing AJ, Ruchaud S, Hudson DF, Honda R, Nigg EA, Gerloff DL, Earnshaw WC. 2004. Borealin: A novel chromosomal passenger required for stability of the bipolar mitotic spindle. J Cell Biol 166: 179-191.

Giet R, Glover DM. 2001. Drosophila aurora B kinase is required for histone $\mathrm{H} 3$ phosphorylation and condensin recruitment during chromosome condensation and to organize the central spindle during cytokinesis. J Cell Biol 152: 669-682.

Giet R, Prigent C. 1999. Aurora/Ipllp-related kinases, a new oncogenic family of mitotic serine-threonine kinases. $J$ Cell Sci 112: 3591-3601.

Glover DM. 2003. Aurora A on the mitotic spindle is activated by the way it holds its partner. Mol Cell 12: 797-799.

Godek KM, Kabeche L, Compton DA. 2015. Regulation of kinetochore-microtubule attachments through homeostatic control during mitosis. Nat Rev Mol Cell Biol 16: 57-64.

Haase J, Bonner MK, Halas H, Kelly AE. 2017. Distinct roles of the chromosomal passenger complex in the detection of and response to errors in kinetochore-microtubule attachment. Dev Cell 42: 640-654.e645.

Hans F, Skoufias DA, Dimitrov S, Margolis RL. 2009. Molecular distinctions between Aurora A and B: A single residue change transforms Aurora A into correctly localized and functional Aurora B. Mol Biol Cell 20: 3491-3502.

Hauf S, Cole RW, LaTerra S, Zimmer C, Schnapp G, Walter R, Heckel A, van Meel J, Rieder CL, Peters JM. 2003. The small molecule Hesperadin reveals a role for Aurora B in correcting kinetochore-microtubule attachment and in maintaining the spindle assembly checkpoint. J Cell Biol 161: 281-294.

Hindriksen S, Lens SMA, Hadders MA. 2017. The ins and outs of Aurora B inner centromere localization. Front Cell Dev Biol 5: 112 .

Hirota T, Kunitoku N, Sasayama T, Marumoto T, Zhang D, Nitta M, Hatakeyama K, Saya H. 2003. Aurora-A and an interacting activator, the LIM protein Ajuba, are required for mitotic commitment in human cells. Cell 114: 585-598.

Honda R, Korner R, Nigg EA. 2003. Exploring the functional interactions between Aurora B, INCENP, and survivin in mitosis. Mol Biol Cell 14: 3325-3341.

Hutterer A, Berdnik D, Wirtz-Peitz F, Zigman M, Schleiffer A, Knoblich JA. 2006. Mitotic activation of the kinase Aurora-A requires its binding partner Bora. Dev Cell 11: 147-157.

Jeyaprakash AA, Klein UR, Lindner D, Ebert J, Nigg EA, Conti E. 2007. Structure of a Survivin-Borealin-INCENP core complex reveals how chromosomal passengers travel together. Cell 131: 271-285.

Joukov V, De Nicolo A, Rodriguez A, Walter JC, Livingston DM. 2010. Centrosomal protein of $192 \mathrm{kDa}$ (Cep192) promotes centrosome-driven spindle assembly by engaging in organelle-specific Aurora A activation. Proc Natl Acad Sci 107: 21022-21027.

Kaitna S, Mendoza M, Jantsch-Plunger V, Glotzer M. 2000. Incenp and an aurora-like kinase form a complex essential for chromosome segregation and efficient completion of cytokinesis. Curr Biol 10: 1172-1181.

Kallio MJ, McCleland ML, Stukenberg PT, Gorbsky GJ. 2002. Inhibition of aurora B kinase blocks chromosome segregation, overrides the spindle checkpoint, and perturbs microtubule dynamics in mitosis. Curr Biol 12: 900-905.

Kapoor TM, Lampson MA, Hergert P, Cameron L, Cimini D, Salmon ED, McEwen BF, Khodjakov A. 2006. Chromosomes can congress to the metaphase plate before biorientation. Science 311: 388-391.

Katayama H, Brinkley WR, Sen S. 2003. The Aurora kinases: Role in cell transformation and tumorigenesis. Cancer Metastasis Rev 22: 451-464.
Katayama H, Sasai K, Kloc M, Brinkley BR, Sen S. 2008. Aurora kinase-A regulates kinetochore/chromatin associated microtubule assembly in human cells. Cell Cycle 7: 2691-2704.

Kettenbach AN, Schweppe DK, Faherty BK, Pechenick D, Pletnev AA, Gerber SA. 2011. Quantitative phosphoproteomics identifies substrates and functional modules of Aurora and Polo-like kinase activities in mitotic cells. Sci Signal 4: rs5.

Khan J, Ezan F, Cremet JY, Fautrel A, Gilot D, Lambert M, Benaud C, Troadec MB, Prigent C. 2011. Overexpression of active Aurora-C kinase results in cell transformation and tumour formation. PLoS One 6: e26512.

Kim Y, Holland AJ, Lan W, Cleveland DW. 2010. Aurora kinases and protein phosphatase 1 mediate chromosome congression through regulation of CENP-E. Cell 142: 444-455.

Klein UR, Nigg EA, Gruneberg U. 2006. Centromere targeting of the chromosomal passenger complex requires a ternary subcomplex of Borealin, Survivin, and the N-terminal domain of INCENP. Mol Biol Cell 17: 2547-2558.

Koch A, Krug K, Pengelley S, Macek B, Hauf S. 2011. Mitotic substrates of the kinase aurora with roles in chromatin regulation identified through quantitative phosphoproteomics of fission yeast. Sci Signal 4: rs6.

Krenn V, Musacchio A. 2015. The Aurora B kinase in chromosome bi-orientation and spindle checkpoint signaling. Front Oncol 5: 225 .

Kufer TA, Sillje HH, Korner R, Gruss OJ, Meraldi P, Nigg EA. 2002. Human TPX2 is required for targeting Aurora-A kinase to the spindle. J Cell Biol 158: 617-623.

Kunitoku N, Sasayama T, Marumoto T, Zhang D, Honda S, Kobayashi O, Hatakeyama K, Ushio Y, Saya H, Hirota T. 2003. CENP-A phosphorylation by Aurora-A in prophase is required for enrichment of Aurora-B at inner centromeres and for kinetochore function. Dev Cell 5: 853-864.

Lampson MA, Grishchuk EL. 2017. Mechanisms to avoid and correct erroneous kinetochore-microtubule attachments. Biology (Basel) 6: pii: E1. doi: 10.3390/biology6010001.

Lens SM, Wolthuis RM, Klompmaker R, Kauw J, Agami R, Brummelkamp T, Kops G, Medema RH. 2003. Survivin is required for a sustained spindle checkpoint arrest in response to lack of tension. EMBO J 22: 2934-2947.

Li S, Deng Z, Fu J, Xu C, Xin G, Wu Z, Luo J, Wang G, Zhang S, Zhang B, et al. 2015. Spatial compartmentalization specializes the function of Aurora A and Aurora B. J Biol Chem 290: 17546-17558.

Liu ST, Rattner JB, Jablonski SA, Yen TJ. 2006. Mapping the assembly pathways that specify formation of the trilaminar kinetochore plates in human cells. J Cell Biol 175: 41-53.

Maddox P, Desai A, Oegema K, Mitchison TJ, Salmon ED. 2002. Poleward microtubule flux is a major component of spindle dynamics and anaphase A in mitotic Drosophila embryos. Curr Biol 12: 1670-1674.

Maddox P, Straight A, Coughlin P, Mitchison TJ, Salmon ED. 2003. Direct observation of microtubule dynamics at kinetochores in Xenopus extract spindles: Implications for spindle mechanics. J Cell Biol 162: 377-382.

Maia AF, Feijao T, Vromans MJ, Sunkel CE, Lens SM. 2010. Aurora B kinase cooperates with CENP-E to promote timely anaphase onset. Chromosoma 119: 405-413.

Marumoto T, Honda S, Hara T, Nitta M, Hirota T, Kohmura E, Saya H. 2003. Aurora-A kinase maintains the fidelity of early and late mitotic events in HeLa cells. J Biol Chem 278: 5178651795 .

Meraldi P, Honda R, Nigg EA. 2004. Aurora kinases link chromosome segregation and cell division to cancer susceptibility. Curr Opin Genet Dev 14: 29-36.

Murata-Hori M, Wang YL. 2002. The kinase activity of aurora B is required for kinetochore-microtubule interactions during mitosis. Curr Biol 12: 894-899.

Murata-Hori M, Tatsuka M, Wang YL. 2002. Probing the dynamics and functions of aurora B kinase in living cells during mitosis and cytokinesis. Mol Biol Cell 13: 1099-1108. 
Musacchio A, Desai A. 2017. A molecular view of kinetochore assembly and function. Biology (Basel) 6: pii: E5. doi: 10.3390/biology6010005.

Nguyen AL, Schindler K. 2017. Specialize and divide (twice): Functions of three Aurora kinase homologs in mammalian oocyte meiotic maturation. Trends Genet 33: 349-363.

Nguyen AL, Gentilello AS, Balboula AZ, Shrivastava V, Ohring J, Schindler K. 2014. Phosphorylation of threonine 3 on histone $\mathrm{H} 3$ by haspin kinase is required for meiosis I in mouse oocytes. J Cell Sci 127: 5066-5078.

Ohashi S, Sakashita G, Ban R, Nagasawa M, Matsuzaki H, Murata Y, Taniguchi H, Shima H, Furukawa K, Urano T. 2006. Phospho-regulation of human protein kinase Aurora-A: Analysis using anti-phospho-Thr288 monoclonal antibodies. Oncogene 25: 7691-7702.

Ozlu N, Srayko M, Kinoshita K, Habermann B, O’Toole ET, Muller-Reichert T, Schmalz N, Desai A, Hyman AA. 2005. An essential function of the C. elegans ortholog of TPX2 is to localize activated aurora A kinase to mitotic spindles. Dev Cell 9: 237-248.

Petersen J, Paris J, Willer M, Philippe M, Hagan IM. 2001. The $S$. pombe aurora-related kinase Ark1 associates with mitotic structures in a stage dependent manner and is required for chromosome segregation. J Cell Sci 114: 4371-4384.

Quartuccio SM, Dipali SS, Schindler K. 2017. Haspin inhibition reveals functional differences of interchromatid axis-localized AURKB and AURKC. Mol Biol Cell 28: 2233-2240.

Santaguida S, Vernieri C, Villa F, Ciliberto A, Musacchio A. 2011. Evidence that Aurora B is implicated in spindle checkpoint signalling independently of error correction. EMBO J 30: $1508-1519$.

Sasai K, Katayama H, Stenoien DL, Fujii S, Honda R, Kimura M, Okano Y, Tatsuka M, Suzuki F, Nigg EA, et al. 2004. Aurora-C kinase is a novel chromosomal passenger protein that can complement Aurora-B kinase function in mitotic cells. Cell Motil Cytoskeleton 59: 249-263.

Sasai K, Katayama H, Hawke DH, Sen S. 2016. Aurora-C interactions with Survivin and INCENP reveal shared and distinct features compared with Aurora-B chromosome passenger protein complex. PLoS One 11: e157305.

Satinover DL, Leach CA, Stukenberg PT, Brautigan DL. 2004. Activation of Aurora-A kinase by protein phosphatase inhibitor-2, a bifunctional signaling protein. Proc Natl Acad Sci 101: 8625-8630.

Schindler K, Davydenko O, Fram B, Lampson MA, Schultz RM. 2012. Maternally recruited Aurora C kinase is more stable than Aurora B to support mouse oocyte maturation and early development. Proc Natl Acad Sci 109: E2215-E2222.

Schumacher JM, Golden A, Donovan PJ. 1998. AIR-2: An Aurora/Ipl1-related protein kinase associated with chromosomes and midbody microtubules is required for polar body extrusion and cytokinesis in Caenorhabditis elegans embryos. J Cell Biol 143: 1635-1646.

Seki A, Coppinger JA, Jang CY, Yates JR, Fang G. 2008. Bora and the kinase Aurora a cooperatively activate the kinase Plk1 and control mitotic entry. Science 320: $1655-1658$.

Sessa F, Mapelli M, Ciferri C, Tarricone C, Areces LB, Schneider TR, Stukenberg PT, Musacchio A. 2005. Mechanism of Aurora $\mathrm{B}$ activation by INCENP and inhibition by hesperadin. Mol Cell 18: 379-391.

Sharif B, Na J, Lykke-Hartmann K, McLaughlin SH, Laue E, Glover DM, Zernicka-Goetz M. 2010. The chromosome passenger complex is required for fidelity of chromosome transmission and cytokinesis in meiosis of mouse oocytes. J Cell Sci 123: 4292-4300.

Shuda K, Schindler K, Ma J, Schultz RM, Donovan PJ. 2009. Aurora kinase B modulates chromosome alignment in mouse oocytes. Mol Reprod Dev 76: 1094-1105.
Tanaka TU, Rachidi N, Janke C, Pereira G, Galova M, Schiebel E, Stark MJ, Nasmyth K. 2002. Evidence that the Ipl1-Sli15 (Aurora kinase-INCENP) complex promotes chromosome biorientation by altering kinetochore-spindle pole connections. Cell 108: 317-329.

Tang CJ, Lin CY, Tang TK. 2006. Dynamic localization and functional implications of Aurora-C kinase during male mouse meiosis. Dev Biol 290: 398-410.

Tatsuka M, Katayama H, Ota T, Tanaka T, Odashima S, Suzuki F, Terada Y. 1998. Multinuclearity and increased ploidy caused by overexpression of the aurora- and Ipl1-like midbody-associated protein mitotic kinase in human cancer cells. Cancer Res 58: 4811-4816.

Terada Y, Tatsuka M, Suzuki F, Yasuda Y, Fujita S, Otsu M. 1998. AIM-1: A mammalian midbody-associated protein required for cytokinesis. EMBO J 17: 667-676.

Tsou JH, Chang KC, Chang-Liao PY, Yang ST, Lee CT, Chen YP, Lee YC, Lin BW, Lee JC, Shen MR, et al. 2011. Aberrantly expressed AURKC enhances the transformation and tumourigenicity of epithelial cells. J Pathol 225: 243-254.

Ulisse S, Delcros JG, Baldini E, Toller M, Curcio F, Giacomelli L, Prigent C, Ambesi-Impiombato FS, D’Armiento M, ArlotBonnemains Y. 2006. Expression of Aurora kinases in human thyroid carcinoma cell lines and tissues. Int J Cancer 119: 275-282.

Vader G, Kauw JJ, Medema RH, Lens SM. 2006. Survivin mediates targeting of the chromosomal passenger complex to the centromere and midbody. EMBO Rep 7: 85-92.

Vader G, Lens SM. 2008. The Aurora kinase family in cell division and cancer. Biochim Biophys Acta 1786: 60-72.

van der Horst A, Lens SM. 2014. Cell division: Control of the chromosomal passenger complex in time and space. Chromosoma 123: 25-42.

Welburn JP, Vleugel M, Liu D, Yates JR III, Lampson MA, Fukagawa T, Cheeseman IM. 2010. Aurora B phosphorylates spatially distinct targets to differentially regulate the kinetochore-microtubule interface. Mol Cell 38: 383-392.

Wheatley SP, Carvalho A, Vagnarelli P, Earnshaw WC. 2001. INCENP is required for proper targeting of Survivin to the centromeres and the anaphase spindle during mitosis. Curr Biol 11: 886-890.

Yang KT, Li SK, Chang CC, Tang CJ, Lin YN, Lee SC, Tang TK. 2010. Aurora-C kinase deficiency causes cytokinesis failure in meiosis I and production of large polyploid oocytes in mice. Mol Biol Cell 21: 2371-2383.

Yang KT, Tang CJ, Tang TK. 2015. Possible role of Aurora-C in meiosis. Front Oncol 5: 178.

Yasui Y, Urano T, Kawajiri A, Nagata K, Tatsuka M, Saya H, Furukawa K, Takahashi T, Izawa I, Inagaki M. 2004. Autophosphorylation of a newly identified site of Aurora-B is indispensable for cytokinesis. J Biol Chem 279: 12997-13003.

Ye AA, Deretic J, Hoel CM, Hinman AW, Cimini D, Welburn JP, Maresca TJ. 2015. Aurora A kinase contributes to a pole-based error correction pathway. Curr Biol 25: 1842-1851.

Zaytsev AV, Sundin LJ, DeLuca KF, Grishchuk EL, DeLuca JG. 2014. Accurate phosphoregulation of kinetochore-microtubule affinity requires unconstrained molecular interactions. J Cell Biol 206: 45-59.

Zaytsev AV, Mick JE, Maslennikov E, Nikashin B, DeLuca JG, Grishchuk EL. 2015. Multisite phosphorylation of the NDC80 complex gradually tunes its microtubule-binding affinity. Mol Biol Cell 26: 1829-1844.

Zeitlin SG, Shelby RD, Sullivan KF. 2001. CENP-A is phosphorylated by Aurora B kinase and plays an unexpected role in completion of cytokinesis. J Cell Biol 155: 1147-1157.

Zhao ZS, Lim JP, Ng YW, Lim L, Manser E. 2005. The GITassociated kinase PAK targets to the centrosome and regulates Aurora-A. Mol Cell 20: 237-249. 


\section{$\$_{\text {CSH }}^{\infty}$ Cold Spring Harbor Symposia SYMPOSIA on Quantitative Biology}

\section{Aurora A Kinase Function at Kinetochores}

Jennifer G. DeLuca

Cold Spring Harb Symp Quant Biol 2017 82: 91-99 originally published online April 26, 2018 Access the most recent version at doi:10.1101/sqb.2017.82.034991

References This article cites 116 articles, 54 of which can be accessed free at: http://symposium.cshlp.org/content/82/91.full.html\#ref-list-1

\section{Creative Commons License}

Email Alerting Service
This article is distributed under the terms of the http://creativecommons.org/licenses/by-nc/4.0/, which permits reuse and redistribution, except for commercial purposes, provided that the original author and source are credited.

Receive free email alerts when new articles cite this article - sign up in the box at the top right corner of the article or click here. 